山्山FANÇAISE

$>\mathrm{DE}$

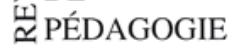

\section{Revue française de pédagogie}

Recherches en éducation

$207 \mid 2020$

La LPPR et la réforme de l'enseignement supérieur et de la recherche: analyses critiques

\title{
La LPPR et la réforme de l'enseignement supérieur et de la recherche : analyses critiques
}

The LPPR and the reform of higher education and research in France: critical analyses

Sylvain Doussot et Xavier Pons

\section{OpenEdition}

Journals

\section{Édition électronique}

URL : https://journals.openedition.org/rfp/9141

DOI : 10.4000/rfp.9141

ISSN : 2105-2913

Éditeur

ENS Éditions

Édition imprimée

Date de publication : 30 juin 2020

Pagination : 11-18

ISBN : 979-10-362-0297-1

ISSN : 0556-7807

Référence électronique

Sylvain Doussot et Xavier Pons, «La LPPR et la réforme de l'enseignement supérieur et de la recherche : analyses critiques », Revue française de pédagogie [En ligne], 207 | 2020, mis en ligne le 30 juin 2020, consulté le 13 janvier 2023. URL : http://journals.openedition.org/rfp/9141 ; DOI : https:// doi.org/10.4000/rfp.9141 


\title{
La LPPR et la réforme de l'enseignement supérieur et de la recherche : analyses critiques
}

\author{
Sylvain Doussot \\ Xavier Pons
}

Le 3 mars 2020, le comité de rédaction de la Revue française de pédagogie a décidé, à une très grande majorité, de s'associer au mouvement de contestation de la Loi de programmation pluriannuelle de la recherche (LPPR) initié dans le monde scientifique depuis, notamment, la mise en grève le 6 janvier 2020 de la revue de sciences sociales Genèses. La manière la plus adéquate de le faire à ses yeux pour une revue de recherche de référence, outre l'affichage systématique en ligne de son soutien solidaire, a été de concevoir un dossier spécial sur le sujet afin d'alimenter le débat public et les réflexions des lecteurs.

\section{Un dossier sous contraintes}

Si cette décision fut relativement facile à prendre, sa mise en œuvre s'est heurtée rapidement à plusieurs contraintes majeures. La première renvoie à l'objet LPPR, dont le contenu véritable était incertain et flou au moment où le dossier fut lancé, ce qui a rendu plus difficile son analyse objective et d'autant plus nécessaire l'inscription de cet objet dans un questionnement plus général sur les transformations contemporaines de l'enseignement supérieur et de la recherche (ESR), en France et ailleurs. Il y avait en effet un risque majeur, en concevant un dossier sur ce thème, de céder aux sirènes de l'actualité, de réifier cet objet, de surestimer son importance et d'interroger les changements institutionnels à l'œuvre au prisme des catégories et des visions qu'en donnent les gouvernants au moment de formaliser leurs programmes d'action publique, plutôt que selon l'évolution des questionnements scientifiques sur le sujet.

La deuxième série de contraintes, particulièrement fortes, renvoie à la gestion de l'épidémie de Covid-19 qui a eu deux effets sur la conception de ce dossier. D’abord, les agendas politique et médiatique se sont concentrés très fortement, voire exclusivement, sur cette crise, reléguant ainsi au second plan l'actualité de la contestation sociale autour de la LPPR d'une part, et la présentation de cette loi au Parlement d'autre part, et ce avant que cette actualité politique ne s'accélère de nouveau brutalement en juin 2020. Un doute a même pesé au cours de la période sur le maintien de 
cette loi à terme, et ce, au moment même où nous sollicitions les auteurs, la ministre de l'Enseignement supérieur, de la Recherche et de I'Innovation Frédérique Vidal déclarant lors d'un point de presse téléphonique le jeudi 19 mars 2020, après les annonces du Président Emmanuel Macron d'une augmentation du budget de la recherche de 5 milliards d'euros, que «l'important, c'est que nous puissions d'ores et déjà garantir et sécuriser le réinvestissement de 5 milliards d'euros [...] qu'il y ait ou non une LPPR validée par le Parlement dans les mois qui viennent ${ }^{1}$. Certains auteurs sollicités pour ce dossier ont ainsi vu dans cette déclaration un langage diplomatique disant implicitement que cette loi ne serait pas votée. Pourquoi alors lui consacrer un dossier? La mention de la LPPR dans la lettre de la ministre adressée à l'ensemble des personnels le 13 mai 2020, les revendications par différents acteurs d'un «plan d'urgence» pour l'ESR à la rentrée 2020 qui ont suivi, tout comme l'actualité politique précipitée de la fin de l'année universitaire ${ }^{2}$, ont cependant confirmé ensuite l'actualité de cette loi qui est présentée au Parlement à I'heure où nous écrivons ces lignes et qui, indépendamment du sort qui lui sera réservé, met le doigt sur des enjeux de fond en matière d'ESR. Second effet : comme pour de nombreuses personnes travaillant dans le secteur de I'ESR, le confinement et la fermeture des établissements, puis leur levée progressive, ont induit de nombreuses activités professionnelles supplémentaires en distanciel, chronophages et nouvelles pour une partie de ces personnes, ce qui a réduit les fenêtres d'écriture des articles dans les agendas professionnels des uns et des autres d'une part, mais aussi la disponibilité d'esprit de certains auteurs sollicités pour se lancer dans une analyse de la LPPR et de ses effets, I'heure étant plutôt à la surcharge cognitive. "Je n'ai toujours pas réussi à m'y mettre et je n'y arrive pas. Mon cerveau n'arrive pas à retourner à ces questions-là, et le temps pour le faire va durablement me manquer : le confinement a plutôt entraîné une forte augmentation de la charge de travail qu'une diminution, tout est devenu plus compliqué, et donc y compris prendre ce temps de réfléchir à ce que nous faisons, et aux conditions dans lesquelles nous le faisons. Malheureusement donc, je dois renoncer, j'en

1 Dépêche $A E F n^{\circ} 624148$ du 19 mars 2020 (nous soulignons).

2 Convocation le 4 juin des syndicats à une réunion multilatérale sur le sujet prévue pour le 12 juin matin avec organisation l'aprèsmidi d'une séance du Conseil national de l'enseignement supérieur et de la recherche, séance qui sera finalement reportée au 18 juin suis profondément désolé», nous a ainsi répondu un collègue ayant au départ accepté de contribuer.

\section{Un dossier hybride}

Face à ces contraintes, le comité de rédaction a fait plusieurs choix. Le premier a été de problématiser le dossier autour de l'analyse des évolutions et des mouvements de réforme plus larges dans lesquels s'inscrit la LPPR afin de ne pas rester prisonnier du sort politique que connaîtra cette loi en particulier et d'inviter les auteurs à se concentrer sur les enjeux de fond que cette séquence révèle en matière de transformation de l'ESR.

Le second choix a été de concevoir un dossier hybride au sens où il ne rassemble pas comme d'ordinaire des articles de recherche uniquement, mais les contributions de spécialistes de l'ESR aux profils variés, dont les contributions peuvent reprendre les codes d'un article de recherche classique ou non ${ }^{3}$, et dont la spécialisation peut reposer soit sur des activités de recherche prenant explicitement les transformations de l'ESR comme objet d'analyse, soit sur des activités et des responsabilités, professionnelles, institutionnelles ou militantes, fournissant un poste d'observation privilégié de celles-ci.

En lien avec les deux choix précédents, mais aussi avec l'esprit de la revue, nous avons enfin demandé aux auteurs de fournir des analyses critiques étayées de leur point de vue, qu'il soit scientifique, expérientiel, militant... II s'est alors agi de trouver un juste équilibre dans le dialogue avec eux entre la prise en compte d'un côté des profils d'article et des points de vue engagés qui pouvaient être exprimés et qui sont tout à fait légitimes pour un dossier de ce type, et de l'autre les besoins d'argumentation et d'objectivation des propos tenus, des diagnostics proposés, des inférences effectuées ou des jugements énoncés. L'analyse critique de la LPPR que propose la Revue française de pédagogie passe donc par la mise en dialogue de points de vue argumentés variés sur ce projet, voire divergents, plutôt que par l'adoption d'une position unique déclinée dans plusieurs textes. Ce dossier se différencie en cela d'autres numéros spéciaux de revue consacrés à la LPPR, même si les analyses proposées d'un numéro à l'autre se rejoignent parfois fortement ${ }^{4}$.

3 Certains articles assument ainsi une part de témoignage d'autres d'analyse conjoncturelle.

4 Voir par exemple certaines contributions au numéro 16 de la Nouvelle Revue du travail publié en 2020 , toutes publiées sous le 


\section{Structure du dossier}

Ce dossier est structuré en trois temps. Les premiers articles visent à donner aux lecteurs les éléments de contexte nécessaires à l'entrée dans la problématique du numéro. Dans leur article sur l'évolution historique du groupe professionnel que forment les enseignantschercheurs, Julien Barrier et Emmanuelle Picard, sociologue et historienne ayant tous deux publié plusieurs recherches sur ce thème (voir par exemple Barrier, 2011, Picard, 2020), proposent ainsi une synthèse des trois principaux clivages internes qui traversent cette profession, pour certains depuis plus de deux siècles : le poids des disciplines, les différentes conditions d'exercice du métier d'universitaire et les nouvelles formes de stratification de la profession induites par les réformes successives depuis les années 1990. Sans entrer dans les détails du projet de LPPR lui-même, ils montrent que la séquence politique autour de ce dernier a pour conséquence paradoxale de «simultanément [...] raviver les clivages internes à la profession", puisque la contestation de ce projet de loi peut prendre des formes différentes selon les disciplines en présence par exemple (position dans l'ensemble moins critique des sciences expérimentales, plus critique de la part des sciences humaines et sociales), et «favoriser un sentiment d'unité autour de la dénonciation d'une dégradation généralisée du métier», la communauté universitaire renouant alors dans cette contestation avec un idéal de collégialité qui ne correspond plus à la réalité de leur métier depuis longtemps. La tension entre unité et division au sein du groupe professionnel des enseignants-chercheurs, tension qu'on retrouve bien sûr dans d'autres pays mais que la LPPR vient exacerber, se caractérise ainsi «par la coexistence, d'un côté, de facteurs qui intensifient la concurrence et individualisent les conditions d'exercice du métier et, d'un autre côté, un ensemble de dispositifs qui maintiennent, bon an mal an, une certaine égalité de traitement et de statut à l'échelle nationale».

L'article d'Anne Mascret, journaliste spécialisée en éducation, procède pour sa part à une analyse conjoncturelle de la réforme LPPR à partir des dépêches publiées par l'agence AEF au sein de laquelle elle suit I'actualité de I'ESR depuis plusieurs années. Après avoir déterminé dans quelle mesure celle-ci se présente

pseudonyme Camille Noûs (numéro disponible en ligne : <https://journals.openedition.org/nrt/6477>, consulté le 23 septembre 2020). comme un nouvel avatar des politiques d'excellence françaises qu'elle a étudiées plus en détail par ailleurs (Mascret, 2015), elle revient sur les différentes séquences de ce mouvement de réforme, depuis les premières annonces plutôt discrètes du gouvernement en février 2019 jusqu'aux premiers débats parlementaires de la rentrée 2020 en passant par l'emballement médiatique de l'hiver 2019 et la crise du Covid-19. Donnant à voir les différentes étapes d'une réforme «en train de se faire», elle interroge la stratégie politique du gouvernement et conclut au gâchis que risque de constituer finalement une réforme contestée qui prévoit pourtant une hausse forte du budget de la recherche.

L'article de Christine Musselin clôt ce premier temps du dossier tout en amorçant le deuxième consacré à l'analyse des éléments constitutifs de ce projet de réforme et à leur philosophie politique d'ensemble. La sociologue se livre d'abord à un état des lieux des principales transformations qui ont affecté I'ESR en France en prenant appui sur de nombreuses enquêtes passées et récentes (par exemple Musselin, 2017). Ces transformations expliquent selon elle la carrière paradoxale de ce projet de loi au contenu incertain qui suscite beaucoup d'oppositions alors qu'il prévoit des hausses budgétaires importantes : la centralisation et la bureaucratisation accrues du fonctionnement des universités, leur plus forte différenciation sous l'effet des évaluations et des financements sur projets, le renforcement d'un pilotage de la recherche et le moindre nombre de postes ouverts au recrutement sont autant d'éléments qui se trouvent renforcés dans le projet de LPPR alors qu'ils sont contestés par une partie importante de la profession universitaire, notamment celle qui ne peut tirer aucun avantage de ces évolutions et voit ses conditions de travail se dégrader. L'auteure regrette ainsi la forte focalisation de ce projet de loi sur le modèle unique de la grande université de recherche internationalisée, focalisation qui laisse de côté d'autres missions (comme l'enseignement et la formation) ou des établissements ayant d'autres stratégies de développement, par exemple locales. Elle regrette aussi que ce projet n'ait pas été l'occasion de réfléchir plus intensément à la gestion de la carrière des enseignants-chercheurs français et formule même quelques pistes de travail pour éviter que ce moment réformateur reste une «occasion manquée». 
L'article de Thibaut Rioufreyt et Camille Noûs ${ }^{5}$ propose quant à lui une analyse des principaux éléments constitutifs de cette loi à partir d'un corpus de documents rassemblant différentes versions du projet de LPPR (y compris les documents officiels publiés par le ministère le 7 juin 2020 à savoir le projet de loi, le rapport annexe et l'exposé des motifs), des dépêches de presse, des rapports publics, des communiqués de presse et des annonces gouvernementales. Après en avoir synthétisé les principales dispositions visant à renforcer le "pilotage stratégique» de la recherche, les financements par projets et/ou privés, l'évaluation des personnels, la dérégulation des statuts, la contractualisation et les partenariats public-privé, les auteurs mettent en évidence comment ces impératifs peuvent à leurs yeux entrer en contradiction avec une activité de recherche scientifique autonome, en limitant le temps de recherche effectif des universitaires, en les incitant à approfondir des thématiques de recherche établies et rentables ou en généralisant le modèle de la recherche et innovation à des activités ou des secteurs dans lesquels celle-ci n'est pas toujours évidente.

Cette perspective est proche de celle adoptée par Philippe Blanchet, sociolinguiste et didacticien des langues qui suit et commente l'actualité de l'ESR depuis de nombreuses années (Blanchet, 2019). L'auteur pointe d'abord la stratégie de communication du gouvernement, ses non-dits et le peu de données publiques sur le contenu du projet de loi avant juin 2020. Après en avoir synthétisé les principaux éléments (précarisation des chercheurs, privatisation de la recherche, impératif de la course aux financements), il interroge le recours à des outils de régulation libéraux pour résoudre les problèmes de l'ESR alors que ceux-ci sont pour lui grandement à l'origine des maux qu'ils entendent combattre. Il termine en rappelant plusieurs revendications d'une partie importante de la communauté universitaire : la stabilité des moyens, l'indépendance statutaire, une production de connaissance scientifique autonome et désintéressée et le respect des personnels dans la gestion de leur carrière.

L'article de Barbara Stiegler et Christophe Pébarthe termine ce deuxième temps du dossier tout en ouvrant

5 Pour ses concepteurs, le collectif RogueESR, Camille Noûs est un «individu collectif qui symbolise [un] attachement profond aux valeurs d'éthique et de probation que porte le débat contradictoire [et qui] est insensible aux indicateurs élaborés par le management institutionnel de la recherche» (voir la description qui en est faite par le laboratoire «délocalisé» Cogitamus disponible sur le troisième qui insiste plus spécifiquement sur les logiques et les principes qui président au projet de LPPR. À partir d'une analyse minutieuse des dispositions prévues dans le projet de loi adopté en Conseil des ministres le 22 juillet 2020 en matière de financement de la recherche - dispositions financières à l'effectivité plus incertaine que ne le laisse entendre l'affichage politique qui en est fait parfois ${ }^{6}-$ les auteurs, I'une philosophe ayant beaucoup publié sur le néolibéralisme, l'autre historien et représentant syndical, mettent en évidence le développement progressif d'un autre type de concurrence que celle proprement scientifique, une concurrence reposant sur des intérêts privés via des financements par projet. Ils pointent les nombreuses conséquences négatives d'un tel mode de financement : le primat de la recherche utile à court terme au nom d'un "solutionnisme technologique »? la disparition de diverses spécialités peu rentables devenant de véritables «disciplines orchidées», ou un financement de la recherche soumis aux problèmes tels qu'ils sont définis dans le débat public plutôt que par rencontre entre des logiques scientifiques et des enjeux et intérêts politiques identifiés, autant de conséquences négatives que la situation sanitaire de 2020 donne clairement à voir en matière de virologie et à laquelle les auteurs font utilement référence.

La question du mode de financement de la recherche est également au centre de l'article de Hugo Harari-Kermadec, économiste de l'éducation attentif aux phénomènes de marchandisation de l'université (Harari-Kermadec, 2019), Mélanie Sargeac, doctorante en sociologie et Camille Noûs. Les auteurs mettent en évidence que la LPPR consacre une logique de « retournement temporel» du financement de la recherche : «au lieu de financer en amont la recherche, comme c'est le cas avec le financement par appels à projet, mais aussi pour les financements récurrents, la LPPR inaugure le financement a posteriori de la recherche, à partir de l'évaluation de ses productions, de même que les frais d'inscription ne sont collectés qu'une fois les inscriptions réalisées » déclarent-ils. Ce retournement temporel, qui s'appuie sur une rationalisation managériale poussée, redéfinit les fondements de l'autonomie académique : I'autonomie corporatiste et scienti-

6 La programmation financière de la recherche jusqu'en 2030 proposée dans le projet de loi, si elle est adoptée, sera par exemple soumise à l'incertitude politique du vote des lois de financement annuel.

7 Les auteurs empruntent cette expression à Evgeni Morozov. 
fique relevant pour une large part d'une activité collective laisse progressivement la place à une autonomie individuelle et budgétaire. Dans ce processus, le développement d'un financement conditionnel de la recherche sur la base d'une évaluation quantitative des productions scientifiques joue un rôle majeur puisque cette évaluation diffuse une représentation quantitative de la valeur de la recherche (caractéristique de l'ère néolibérale pour les auteurs) et qu'elle conduit à une polarisation et une précarisation de celle-ci.

Si plusieurs articles de ce dossier convergent vers une lecture critique de la philosophie néolibérale à I'origine de la LPPR, cette critique ne constitue pas cependant la seule grille de lecture des enjeux liés à la LPPR et à travers elle, à la réforme de I'ESR en France aujourd'hui. Comme le souligne I'article de Christine Musselin, la séquence de réforme actuelle, et plus globalement la philosophie politique qui la caractérise, laissent de côté bon nombre d'autres enjeux majeurs que la seule question du passage à une logique de performance.

C'est le sens de l'article de François Vatin, sociologue du travail ayant à plusieurs reprises analysé les transformations de I'ESR français, que de mettre l'accent sur l'un de ces enjeux de fond que les réformes conjoncturelles comme la LPPR permettent rarement de prendre en compte, à savoir l'évolution du rapport des enseignants-chercheurs à leurs missions. Partant d'un sentiment répandu chez plusieurs de ses collègues de faire «deux métiers qui tendent à s'éloigner de plus en plus l'un de l'autre», sentiment qu'il ne partage pas, l'auteur revient sur la construction du statut d'enseignant-chercheur. II montre les tensions qui traversent ce statut aujourd'hui avec, d'un côté, une dévalorisation matérielle et symbolique des fonctions d'enseignement liée à la massification des universités et à l'évolution des dispositions des étudiants qu'elles accueillent à l'égard des études universitaires et, de l'autre, la valorisation trompeuse d'une mission de recherche au détriment de l'enseignement, par les enseignants-chercheurs eux-mêmes, mais aussi par I'institution qui propose des dispositifs d'aménagement des charges d'enseignement : trompeuse car elle condamne les enseignants-chercheurs à être vainement comparés à des chercheurs de plein statut, trompeuse aussi car elle repose sur une métrologie bien incapable de mesurer précisément les temps de recherche, trompeuse enfin car elle repose sur une vision fausse de l'« université » que l'auteur a combattue tout au long de sa carrière. Dans le prolongement d'analyses antérieures (Vatin \& Vernet, 2009; Vatin, 2016), il pointe alors le «compromis bancal et pervers» qui caractérise la situation actuelle des universités et que la LPPR, comme d'autres réformes conjoncturelles, ne semble pas vouloir trancher : celui de maintenir face à de nouveaux publics d'étudiants ayant pour beaucoup connu un tri scolaire négatif, n'ayant pas un grand appétit pour les études proprement «universitaires» et aspirant à s'insérer efficacement sur le marché du travail, des enseignants-chercheurs qui ont d'autres aspirations professionnelles que de leur enseigner les contenus de formation prévus et qui de ce fait, souffrent au travail.

\section{Incrémentalisme, néolibéralisme et professionnalisme}

Si ces analyses sur un objet empirique au sort encore incertain au moment où nous écrivons ces lignes - la LPPR - ne sauraient épuiser les réflexions sur les transformations en cours de l'ESR en France, elles ont le mérite, en plus de leurs qualités intrinsèques, de donner à lire plusieurs dynamiques transversales. Nous en distinguons trois pour terminer cette introduction, sans prétention d'exhaustivité.

La première renvoie aux stratégies de réforme de I'ESR en France que le cas particulier du projet de LPPR, au départ discret et faussement consensuel, puis âprement contesté, possiblement suspendu avant d'être de nouveau poursuivi à un rythme politique soutenu, donne bien à voir. À la lecture des articles, la stratégie gouvernementale actuelle semble être celle de l'incrémentalisme pragmatique qui caractérise une partie des politiques d'éducation mises en œuvre aujourd'hui (Pons, 2020). Ce terme renvoie à un processus bien identifié en analyse des politiques publiques selon lequel les décideurs, fondamentalement en situation de rationalité limitée, procèdent à une succession d'ajustements de l'existant au nom de ce qui « marche», de ce qui est jugé "efficace» avec une volonté de se situer, à leurs yeux en tout cas, au-delà des clivages idéologiques classiques. Ces ajustements donnent souvent lieu à une «institutionnalisation du flou» (Dubois, 1999) qui rend difficile la compréhension à I'instant t du schéma d'ensemble de la réforme, de son contenu et des valeurs et idéaux qui la sous-tendent. Les auteurs de ce dossier insistent ainsi à plusieurs reprises sur les contenus incertains du projet de loi finalement proposé à l'été 2020, sur l'opacité qui 
entoure le contenu précis de la concertation engagée en 2019, sur le fait que celle-ci se traduira sans doute par une loi-cadre courte dont le contenu détaillé sera précisé ensuite par décrets ${ }^{8}$, sur la nécessité pour en comprendre le contenu et les enjeux de mobiliser un corpus de documents variés et pas toujours faciles d'accès comme le rapport non public sur le «Pilotage de la masse salariale des universités» rédigé par l'Inspection générale des finances et l'Inspection générale de l'administration de l'Éducation nationale et de la Recherche, révélé par I'AEF en février $2020^{\circ}$.

Cet incrémentalisme pragmatique semble d'autant plus efficace que les articles suggèrent l'existence d'une forte codification de la parole publique légitime sur I'ESR. Cette codification apparaît, comme dans d'autres domaines, dans le foisonnement d'acronymes et de dispositifs auxquels il semble impossible d'échapper dès lors qu'on souhaite entrer dans le détail de l'analyse des dispositions mises en œuvre et de leurs effets. Elle est une manifestation évidente de la complexification de l'action publique et d'une rationalisation managériale. Elle est de toute évidence aussi l'instrument d'une domination symbolique du champ de la réforme par des acteurs de I'ESR qui maîtrisent ces acronymes et ces dispositifs pour les promouvoir ou les mettre en œuvre. Elle est enfin une manière de masquer, sous couvert d'un vocable technique froid et prétendument rationnel, une parole plus explicite et plus directe sur les transformations souhaitables. L'exemple de la tribune d'Antoine Petit, présidentdirecteur général du CNRS, publiée dans Les Échos le 26 novembre 2019 et reprise par plusieurs auteurs de ce dossier, soit pour pointer le rôle de catalyseur de la contestation qu'elle a de fait joué, soit pour asseoir une réflexion critique, l'illustre bien. Si cette tribune, dans son ensemble, est plutôt classique pour ce type d'exercice (discours mobilisateur généraliste, appel aux forces scientifiques du pays, références à des grandes personnalités scientifiques et à leurs inventions), il aura suffi d'une phrase $\mathrm{e}^{10}$, et au sein de cette dernière de quelques adjectifs («inégalitaire» et «darwinienne»

8 Au moment où nous écrivons ces lignes, le projet de loi est en première lecture à l'Assemblée nationale, les premiers articles donnant lieu à l'examen de plusieurs centaines de propositions d'amendements.

9 Dépêche AEF nº20974 du 4 février 2020.

10 «ll faut une loi ambitieuse, inégalitaire - oui, inégalitaire, une loi vertueuse et darwinienne, qui encourage les scientifiques, équipes, laboratoires, établissements les plus performants à l'échelle notamment) pour susciter la polémique, repolitiser le débat public sur ce projet de loi et mettre à mal une stratégie de gouvernement incrémentale-pragmatique, révélant ainsi en creux la forte codification de la parole à l'œuvre jusque-là'1 .

Cet incrémentalisme pragmatique enfin a pour conséquence de rendre particulièrement difficile l'examen critique de la réforme, puisque celle-ci n'est jamais présentée explicitement dans son entièreté avant sa validation politique finale (et si possible rapide), et donc de rendre potentiellement caduques les critiques qui anticiperaient trop sur les développements à venir ou qui chercheraient les fondements axiologiques ou idéologiques de ladite réforme. La question s'est posée avec acuité pour la Revue française de pédagogie au moment même de constituer un tel dossier sur la LPPR : le doute laissé par le gouvernement sur la présentation ou non de cette loi au Parlement après l'épisode du Covid-19 implique-t-il que la LPPR soit déjà un objet d'investigation du passé? Répondre oui et considérer qu'il n'y a plus matière à débat sur ce point, n'est-ce pas consacrer l'efficacité d'une stratégie incrémentale-pragmatique et manquer de clairvoyance? Répondre non et continuer à analyser une mobilisation contre un projet de loi potentiellement suspendu, n'est-ce pas refuser de voir une évidence factuelle et faire preuve de corporatisme universitaire? On voit bien que face à une telle stratégie de gouvernement, les répertoires d'action collective d'une profession universitaire par ailleurs plus clivée qu'on ne l'imagine, voire atomisée en période de Covid-19 puis accaparée par les impératifs pédagogiques d'une rentrée universitaire 2020 placée sous l'impératif d'adaptation pédagogique au «distanciel», s'en trouvent bouleversés.

La deuxième dynamique qui apparaît de manière transversale dans plusieurs contributions à ce dossier est la critique du néolibéralisme que le projet de LPPR, comme d'autres lois et politiques avant lui, et ce, quelles que soient les majorités politiques au pouvoir, confirme aux yeux de certains auteurs. De fait, cette

11 Cette mise à mal pourrait cependant s'avérer toute relative dans un contexte de hausse importante des financements octroyés à la recherche dans le cadre du plan de relance de l'économie annoncé en septembre 2020 : le gouvernement souhaite en effet consacrer plusieurs milliards à la recherche (universitaire notamment), ce qui pourrait considérablement changer la structure d'incitations mise en place par la LPPR, la pression à la course aux financements pouvant être paradoxalement plus forte encore en contexte de hausse budgétaire. 
gouvernementalité néolibérale se déploie par un grand nombre de canaux : mise en concurrence des établissements et des unités de recherche, marchandisation de la connaissance et rapport utilitaire à la production scientifique, développement de la quantification, rationalisation managériale et avec elle bureaucratisation de l'ESR... Ce déploiement n'est pas sans lien d'ailleurs avec la stratégie de gouvernement précédemment évoquée, l'incrémentalisme pragmatique étant souvent une stratégie de gouvernement mise en œuvre au nom d'une idéologie (par exemple néolibérale) qui ne peut dire son nom tant elle reste minoritaire dans le secteur d'action publique concerné ou «honteuse $»^{12}$ pour une partie des acteurs. II est frappant en outre de constater combien cette logique, telle qu'elle se déploie en France, semble peu compatible avec la pratique d'une recherche scientifique autonome d'une part, et avec le développement d'un ESR équilibré et répondant aux enjeux de fond d'autre part : le raccourcissement du temps effectivement consacré à la recherche; l'horizon de court terme de la production scientifique qu'impliquent le financement sur projet, la marchandisation, la mise en concurrence mais aussi le lien de plus en plus régulier effectué entre recherche et innovation; l'usure mentale suscitée par la bureaucratisation; le sort réservé aux «vaincus» du pilotage par la performance, qu'il s'agisse des enseignants-chercheurs, des étudiants ou des établissements qui les accueillent, sont autant d'effets négatifs produits par cette vision néolibérale de I'ESR pour plusieurs auteurs de ce dossier.

12 Cet adjectif fait référence au «libéralisme honteux» qui caractérise une partie des classes moyennes en matière de choix de l'école selon Agnès van Zanten (2009).

\section{Bibliographie}

BARRIER J. (2011). «La science en projets : financements sur projet, autonomie professionnelle et transformations du travail des chercheurs académiques ». Sociologie du travail, $\mathrm{n}^{\circ}$ 53(4), p.515-536.

BARRIER J. \& MUSSELIN C. (2015). «La réforme comme opportunité professionnelle : autonomie des établissements et montée en puissance des cadres administratifs des universités». Gouvernement et action publique, n4(4), p.127-151.

BLANCHET P. (2019). Main basse sur I'Université. Paris : Textuel.

DUBOIS V. (1999). La politique culturelle : genèse d'une catégorie d'intervention publique. Paris : Belin.
La dernière dynamique est celle des transformations du professionnalisme universitaire qu'un détour par une réflexion sur les réformes de l'ESR permet d'interroger. Ces réformes, et les contestations qu'elles peuvent susciter, mettent en effet à nu des professionnalités universitaires en forte tension. Ces réformes ne produisent pas les mêmes effets sur un groupe professionnel bien plus clivé qu'on ne l'imagine comme le rappellent Julien Barrier et Emmanuel Picard, certains segments de ce groupe professionnel tirant plus avantage des réformes que d'autres. La succession de ces réformes depuis les années 1990 accentue ainsi la polarisation de ce groupe professionnel avec d'un côté un recours accru à des personnels contractuels et/ou en situation précaire (Harroche, 2019) et de l'autre l'avènement de nouvelles «élites» universitaires par des canaux variés : pouvoir accru des équipes de direction des universités, professionnalisation progressive des métiers de l'encadrement universitaire (Barrier \& Musselin, 2015), investissement dans des missions d'évaluation de la recherche, etc., polarisation que semblent entériner les réflexions ouvertes à la rentrée 2019 sur la possible mise en œuvre d'un système proche de celui des tenure tracks aux États-Unis.

Sylvain Doussot

Université de Nantes, INSPE, CREN sylvain.doussot@univ-nantes.fr

Xavier Pons

Université Paris-Est-Créteil-Val-de-Marne, Lirtes, OSC-Sciences Po xavier.pons@upec.fr

HARARI-KERMADEC H. (2019). Le classement de Shanghai. L'université marchandisée. Paris : Éd. Le Bord de l'eau.

HARROCHE A. (2019). "Les petites mains de l'excellence. Place et rôle des chargées de projet dans la mise en œuvre d'une Initiative d'excellence». Revue française d'administration publique, vol. 169, $\mathrm{n}^{\circ} 1$, p. 151-167.

MASCRET A. (2015). Enseignement supérieur et recherche en France : une ambition d'excellence. Paris : La Documentation française.

MUSSELIN C. (2017). La grande course des universités. Paris : Presses de Sciences Po.

PICARD E. (2020). La profession introuvable? Les universitaires français de l'Université impériale aux universités 
contemporaines. Mémoire d'HDR, Université Paris 1 Panthéon Sorbonne.

PONS X. (2020). «Qu'est-ce qu'une réforme éducative réussie? Une réflexion sociologique». Revue internationale d'éducation, n85, p.33-43.

van ZANTEN A. (2009). Choisir son école. Paris : PUF.
VATIN F. (2016). «Une crise sans fin. L'État, l'enseignement supérieur et les étudiants ». Le Débat, n 192(5), p. 154-172.

VATIN F. \& VERNET A. (2009). «La crise de I'Université française : une perspective historique et socio-démographique». Revue du MAUSS, n³3(1), p. 47-68. 